\title{
Primary Malignant Melanoma of Mandibular Gingiva with Cerebral Metastasis - A Case Report with Review of Literature
}

\author{
Mahalingam Gayathri ${ }^{1}$, Santiago Gnanadeepam, MDS $^{2 *}$, Capt P Raghu ${ }^{3}$, Packiaraj Selvajothi ${ }^{4}$, Muthuponnus Wamy \\ Sumathy ${ }^{2}$, Subramanian Kuzhali ${ }^{4}$, J Sophia Jebapriya ${ }^{4}$ \\ ${ }^{1}$ Postgraduate Student, Department of Oral Medicine\& Radiology, Tamil Nadu Government Dental College and Hospital, Chennai-600003, Tamil \\ Nadu, India \\ ${ }^{2}$ Associate Professor, Department of Oral Pathology, Tamil Nadu Government Dental College and Hospital, Chennai-600003, Tamil Nadu, India \\ ${ }^{3}$ Senior Assistant Professor, Department of Oral Medicine\& Radiology, Tamil Nadu Government Dental College and Hospital, Chennai-600003, Tamil \\ Nadu, India \\ ${ }^{4}$ Assistant Professor, Department of Oral Pathology, Tamil Nadu Government Dental College and Hospital, Chennai-600003, Tamil Nadu, India
}

DOI: $10.36348 /$ sjpm.2020.v05i01.004

| Received: 13.01.2020 | Accepted: 21.01.2020 | Published: 30.01 .2020

*Corresponding author: Santiago Gnanadeepam

\section{Abstract}

Pigmented lesions encountered in routine clinical practice are mostly asymptomatic and benign in nature. However, rarely, a pigmented lesion can be a sign of malignancy. Primary oral mucosal melanomas are rare and aggressive malignancies. At the time of diagnosis most melanomas are in an advanced stage due to early asymptomatic nature. The prognosis remains poor despite treatment. We report a rare case of cerebral metastasis, from primary malignant melanoma of the mandibular gingiva in a 70-year-old male patient who eventually died before initiating the treatment.

Keywords: Primary Malignant Melanoma, Mandibular Gingiva, Cerebral Metastasis, Regional Metastasis.

\begin{abstract}
Copyright @ 2020: This is an open-access article distributed under the terms of the Creative Commons Attribution license which permits unrestricted use, distribution, and reproduction in any medium for non-commercial use (NonCommercial, or CC-BY-NC) provided the original author and sources are credited.
\end{abstract}

\section{INTRODUCTION}

Primary malignant melanoma of oral cavity is exceedingly rare and highly aggressive tumor of melanocytic origin[1].Melanocytes arecells derived from the neural crest that constitute the melanin pigment in the basal layer of epithelium [2]. It was first described by Weber in 1859 [3]. Oral malignant melanoma (OMM) is believed to account for $0.5 \%$ of all malignant oral neoplasms [4] and represents $0.2-$ $8.0 \%$ ofall melanomas $[1,3,4]$. OMM usually occurs in the fourth to sixth decades of life with male predilection (Male-Female ratio of 2:1) [5]. The etiopathogenesis of OMM is poorly understood [6], in contrast to the known risk factors for cutaneous melanoma such as sun exposure, family history; such risk factors have not yet been established for mucosal melanomas [7]. OMM may be primary or metastatic. In the oral cavity most commonly involved sites are hard palate and maxillary alveolar gingiva. OMM are highly aggressive and have tendency to invade the surrounding tissues with early regional and distant dissemination with high rates of treatment failureleading to poorer prognosis than its cutaneous counterpart $[2,8]$. In general, the five-year survival rate ranges from $10 \%$ to $25 \%$ [2].
Here we report a case of primary malignant melanoma of mandibular gingiva with regional and cerebral metastasis. To the best of our knowledge there were no documented cases of cerebral metastasis in Indian literature, fromprimary melanoma of mandibular gingiva.

\section{CASE REPORT}

A 70-year-old male patient was referred from Department of Geriatrics, Government General Hospital to Tamil Nadu Government Dental Collegeand Hospital with a complaint of gradually increasingswelling and growth of one month durationin the right lower jaw. There was no history of pain, bleeding or any discharge from the mass. History of weight loss, loss of appetite, unsteadiness and dizziness was present. The past medical and surgical history was not contributory. Personal history revealedthe habit of heavy tobacco use ( $>25$ cigarettes per day over 30 years).

Neurological examination revealed, swaying to right side when standing on both legs, gait small steps, unsteady and speech disturbances.Extra-oral examination revealed localized swelling in theright submandibular region of size approximately $3 \times 2 \mathrm{~cm}$ with 
smooth surface andcolour of skin appeared normal. On palpation, the right sub-mandibular lymph nodes were hard, fixed and tender. Intraoral examination revealed an exophytic growth in the right alveolar gingiva of size approximately $8 \times 3 \mathrm{~cm}$ extending posteriorly up to 48 region, anteriorly crossing midline to 35 region, medially the lingual alveolar gingiva, laterally buccal alveolar gingiva, inferiorly floor of mouth and buccal vestibule and superiorly uptoocclusal surface of teeth. It was well defined with broad base, irregular margins, blue-black in colour; surface appeared nodular with no secondary changes. On palpation the growth was soft to firm in consistency, tender, compressible, nonblanching, non-pulsatile with bleeding on probing. Grade III mobility was noted in 4546 47, 4443 and 48 (Figure-1).

Based on the clinical findings, a provisional diagnosis of malignant melanoma was made. Differential diagnosis of any vascular related disorders and Kaposi's sarcoma was suspected. The patient underwent investigations such as aspiration, haematological \& urine examinations, HIV screening test and radiological examination. Aspiration was negative. Haematological and urine examinations did not reveal any significant findings. HIV test was nonreactive. Results of the liver and renal function tests were within normal limits. Fine needle aspiration cytology (FNAC) of the rightsub-mandibular lymph node was done followed by incision biopsy of the lesion.

Radiological examination with intraoral periapical radiograph (Figure 2A) and dental panoramic radiograph revealed erosion of right alveolus with irregular borders in relation to 46,47 regions and resorption of roots of 46 and mesial root of 47 was noted (Figure 2B). CBCT revealed osteolytic lesion extending from 44 to 48 region. Erosion involving right alveolus and body of mandible with loss of buccal and lingual cortex and resorption of roots of 46 and mesial root of 47 was noted (Figure 2C). CT scan of chest, abdomen and brain was taken to rule out dissemination and distant metastasis. CT brain revealed hyper-dense lesion in the right frontal and parietal lobe (Figure 2D). FNAC of rightsub-mandibular lymph node was suggestive of metastatic deposits from melanoma.

An incisional biopsy of the primary lesion was performed under local anaesthesiaand sent for histopathological examination which revealed an infiltrative growth pattern of atypical melanocytes in the form of sheets and islands in the connective tissue (Figure 3A, B). The neoplastic cells were large epithelioid like cells with pleomorphic hyperchromatic nuclei, few abnormal mitosis and abundant brownblack pigment in the cytoplasm of the neoplasic cells. The histopathology report was given as malignant melanoma. Further to confirm, the tissue was immunohistochemically stained for HMB-45, a specific marker for melanocytes which revealed intense cytoplasmic positivity of malignant melanocytes for the antibody (Figure $3 \mathrm{C}, \mathrm{D}$ ).

The patient was staged as T4a N1 M1 (Stage IVC) and referred to Department of Oncology for management.Considering the age of patient, extensive size of intraoral lesion combined with cerebral metastasis, a palliative therapy was planned, but the patient died before the treatmentwas initiated.

Table-1: Summary of reported cases of malignant melanoma with cerebral metastasis

\begin{tabular}{|c|c|c|c|c|}
\hline \multirow[t]{2}{*}{ Study } & Age & \multirow{2}{*}{$\begin{array}{l}\text { Primary lesion } \\
\text { Location }\end{array}$} & \multirow[t]{2}{*}{ Metastasis } & \multirow[t]{2}{*}{ Survival } \\
\hline & $/ \mathrm{Sex}$ & & & \\
\hline Martinez EA et al., 2005 [12] & $75 / \mathrm{M}$ & $\begin{array}{l}\text { Hard palate, } \\
\text { maxillary gingiva }\end{array}$ & $\begin{array}{l}\text { Submandibular node, Left } \\
\text { parietal lobe }\end{array}$ & $\begin{array}{l}\text { Died before } \\
\text { treatment }\end{array}$ \\
\hline Gauwerky KJ et al., 2010 [7] & $48 / \mathrm{F}$ & Maxillary gingiva & Right frontal lobe & 6 weeks \\
\hline Tanaka N et al., 2004 [4] & 69/M & Maxillary gingiva & Brain & 8 months \\
\hline Tlholoe MM et al., 2015 [9] & $56 / \mathrm{F}$ & $\begin{array}{l}\text { Maxillary gingiva, } \\
\text { palate }\end{array}$ & Lung, liver and brain & $\begin{array}{l}\text { Died before } \\
\text { treatment }\end{array}$ \\
\hline $\begin{array}{l}\text { Sun CZ et al., } 2012 \text { [15] [1 among } \\
51 \text { cases studied between } 1976 \text { to } \\
2005]\end{array}$ & - & & Brain & - \\
\hline Kridis WB et al., 2016 [14] & $55 / \mathrm{M}$ & $\begin{array}{l}\text { Mandibular gingiva } \\
\text { to tonsillar region }\end{array}$ & $\begin{array}{l}\text { Frontal, occipital lobes, } \\
\text { bilateral pulmonary region }\end{array}$ & 3 months \\
\hline Present case & $70 / \mathrm{M}$ & $\begin{array}{l}\text { Right mandibular } \\
\text { gingiva }\end{array}$ & $\begin{array}{l}\text { right frontal and parietal } \\
\text { lobes, rightsub-mandibular } \\
\text { lymph node }\end{array}$ & $\begin{array}{l}\text { Died before } \\
\text { treatment }\end{array}$ \\
\hline
\end{tabular}




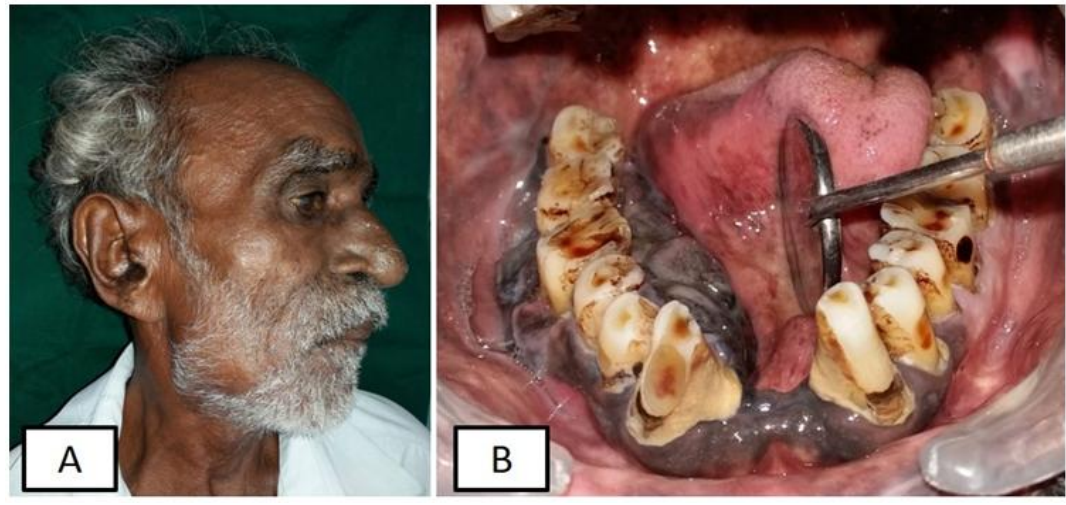

Fig-1: A-Extra oral view shows swelling involving right submandibular region and body of mandible. B- Intraoral view shows bluish black pigmented exophytic growth on right posterior mandibular alveolar gingiva crossing midline and extending to left premolar region

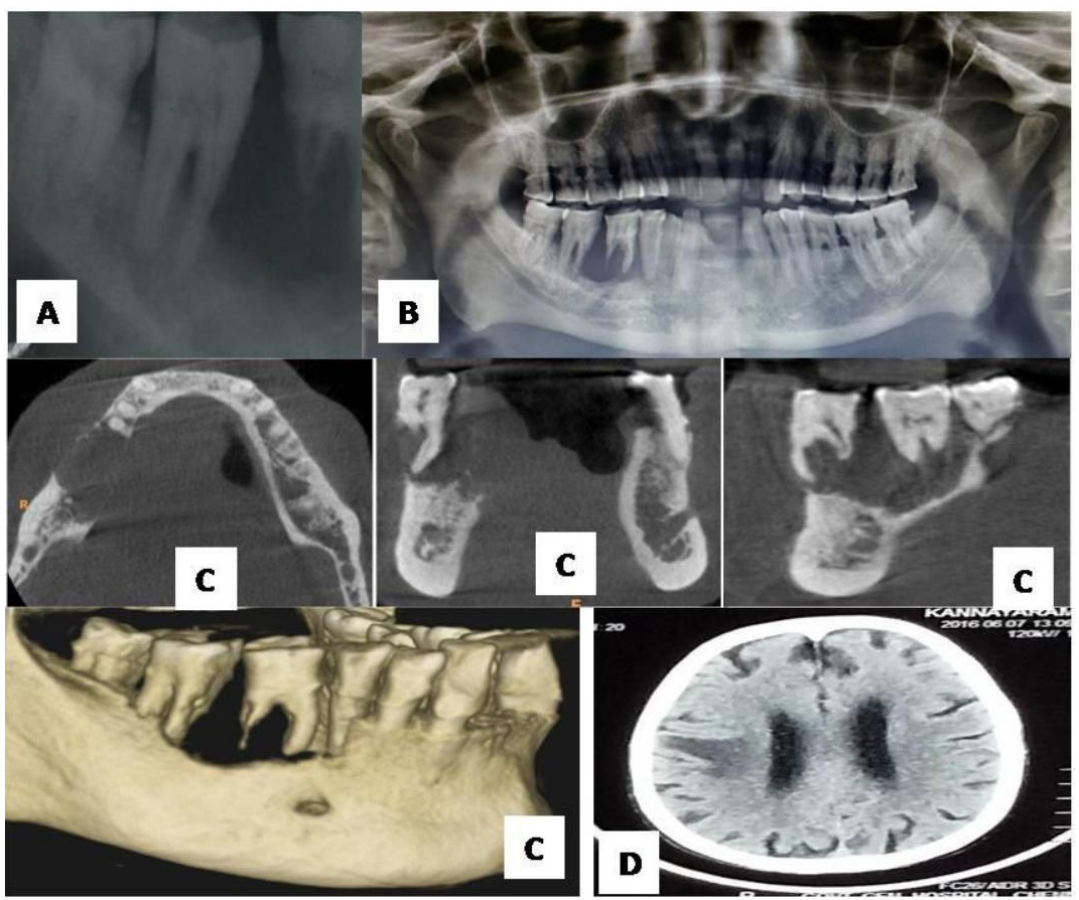

Fig-2: A-Intraoral periapical radiograph reveals erosion of Right mandibular alveolus with extensive resorption of distal root of 46. B: Dental panoramic view reveals erosion of right mandibular alveolus in relation to 46,47 region with resorption of roots and floating teeth appearance. C: CBCT axial, coronal, sagittal view. 3D reconstruction view reveals osteolytic lesion with erosion of alveolus, loss of buccal and lingual cortex and resorption of roots of $\mathbf{4 6}$ and floating teeth appearance. D: CT scan of Brain reveals hypointense lesion involving the frontal and parietal lobes of cerebrum.

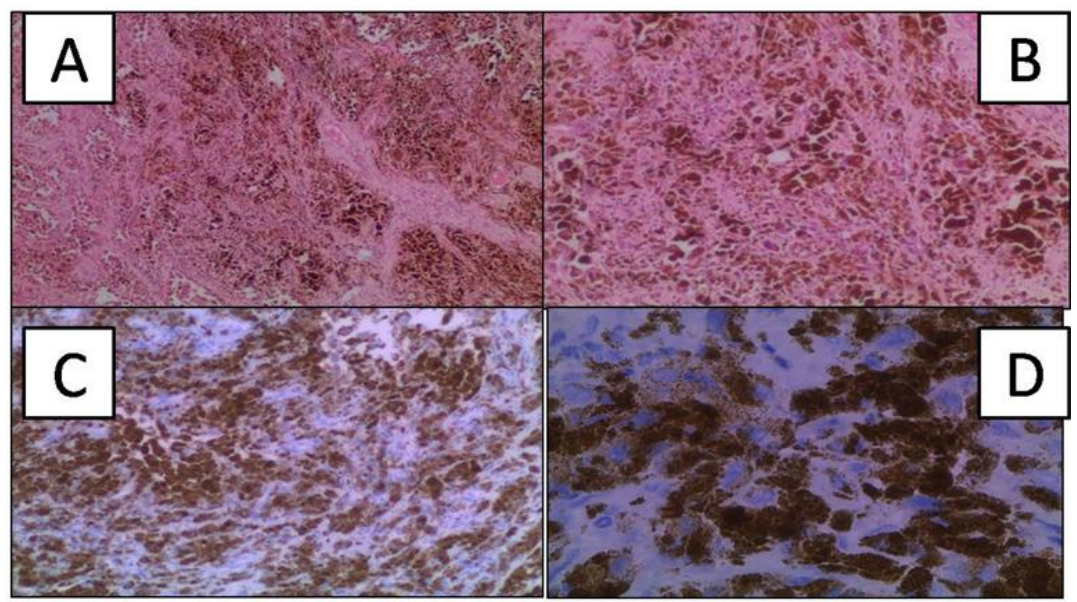

Fig-3: An infiltrative growth pattern in the form of atypical melanocytes with abundant brown- black pigments. (A- H\&E X 200 magnification, B- H\&E X 400 magnification). Cytoplasmic positivity of malignant melanocytes for the HMB-45 antibody. (C-X 200 magnification, D- X 400 magnification) 


\section{DISCUSSION}

Oral mucosal melanoma is a relatively rare malignancy with an aggressive clinic-pathological behavior [9]. Mucosal melanoma is a proliferation of atypical melanocytes at the interface between the epithelium and connective tissue.OMM is believed to arise from pigmented nevi or de novo from apparently normal mucosa. In the oral cavity, mechanical trauma from denture irritation and tobacco use has been as cited possible causative factors with no evidence to support these theories $[1,2,6]$. c-KIT mutations are more likely implicated in the oncogenic process of acrolentiginous and mucosal melanomas [7, 8].

Clinical presentation of melanoma can vary tremendously. There is a male predilection, and affected persons are usually in their fifth through seventh decades of life.Approximately $70 \%$ to $80 \%$ of oral melanomas are found on the hard palate or maxillary alveolusfollowed by mandibular gingiva, tongue, buccal mucosa, and upper and lower lip in the decreasing order [3]. The most common form of oral melanoma is acral lentiginous melanoma.The initial presenting symptom is pigmented swelling [1]. The lesion typically begins as a brown to black macule with irregular borders or show a vascular appearance of grey, purple and red shade or depigmentations [1]. The macule extends laterally, and a lobulated, exophytic mass develops once vertical growth is initiated. Oral melanoma is often reported nodular at the time of diagnosis and relatively soft to palpation. Surface may be smooth or ulcerated. Pain is uncommon except in ulcerated lesions.Other presenting signs and symptoms include bleeding, illfitting dentures, increased mobility of teeth and delayed healing of extraction sockets and regional lymphadenopathy may be present [1]. Our case was a male patient in his seventh decade of life presented with soft non-tender blue black pigmented exophytic nodular growth involving the mandibular alveolar gingiva with bleeding on probing, mobility of teeth and regional lymphadenopathy.

Unlike the cutaneous melanoma, there is no well-defined clinical and pathological classification or diagnostic criteria present for mucosal melanoma. Based on clinical appearance, Tanaka et al., identified five types of OMM: pigmented nodular, non pigmented nodular, pigmented macular, pigmented mixed and nonpigmentedmixed [1]. Greene et al., proposed three criteria for the diagnosis of primary oral melanoma: demonstration of malignant melanoma in the oral mucosa; presence of so-called 'junctional activity' (i.e., melanocytes arranged along the basal layer of the surface epithelium) in the lesion; inability to show malignant melanoma at any other primary site $[2,10]$.

The ABCDE checklist (Asymmetry, Border irregularities, Color variegation, Diameter $>6 \mathrm{~mm}$, and Elevation), widely used in the clinical evaluation of suspicious pigmented cutaneous lesions, could also be used to differentiate OMMs from other oral pigmentations [8]. As for this checklist, the present case has an asymmetrical; irregular borders; dark black color; $8.0 \times 3.0 \times 2.0 \mathrm{~cm}$ in dimension and an elevated surface.

Radiological examination could be useful for evaluation of primary tumour and regional or distant metastases [1]. Radiographically adjacent bone may show evidence of irregular or "moth-eaten" destruction. In our case alveolar bone destruction with resorption of roots and cerebral metastasis was detected.

Histologically it is characterized by the proliferation of atypical melanocytes, larger than normal melanocytes and having varying degree of nuclear pleomorphism and hyperchromatism, with wide variety of shapes, located along the junction between the epithelial and the connective tissue, as well as invading the connective tissue [5]. Malignant cells of OMM show a wide range of shapes, including spindle, plasmocytoid, clear cell and epithelioid ones [1]. Histopathologically, MMs can show three patterns including in-situ pattern (15\%) in which the neoplasm is limited to the epithelium and epithelial - connective tissue interface (junctional), an invasive pattern or nodular pattern $(30 \%)$ in which the neoplasm is found within the underlying connective tissue, and a combined pattern $(55 \%)$ which is invasive melanoma with an in-situ component that is typical in most advanced lesions. Our case showed features of combined pattern. A histological melanoma is confirmed by the Fontana- Masson silver stain and the appropriate immunohistochemical staining pattern that includes S- 100, HMB45 antibodies, Melan - A, tyrosinase and antimicropthalmia transcription factor. For the present case, HMB 45 stained sections confirmed melanoma.

Pigmented lesions of the oral cavity should be viewed with suspicion. It must be differentiated from other forms of pigmented oral diseases, including amalgam tattoo, drug induced or smoking-associated melanosis, physiologic pigmentation, oral melanotic macule, melanocytic nevus and melanoacanthoma, Kaposi's sarcoma, Peutz- Jeghers syndrome, Addison disease $[2,6]$.

Cervical lymph node metastasis most often is evident at initial presentation.In various studies the rate of distant metastases at diagnosis is $10 \%$ to $50 \%$. The lungs are the most frequent site of metastasis, followed by the liver, bones, and brain [8]. Head and neck melanoma metastasize through all the three routes. Cerebral metastasis usually occurs by hematogenous route as it is devoid of lymphatic drainage [13] as noted in our case. 
Risk factors associated with brain metastasis include male gender, mucosal or head and neck primaries, thick or ulcerated neoplasms, acral lentiginous or nodal lesions, and stage IV disease. Kridis WB et al., have reported the first case of primary gingival melanoma in Tunisian literature, having cerebral and pulmonary metastasis [14]. In our case the following risk factors were present and metastasis to regional submandibular node was confirmed by FNAC and metastasis to frontal and parietal lobes by CT brain. Only very few cases have been documented in literature with metastasis to brain from oral cavity (maxillary gingiva and hard palate) by Tanaka $\mathrm{N}$ et al., [4], Martinez EA et al., (2005) [12], Gauwerky KJ et al., [7], Sun CZ et al., 2012 [15], Tlholoe MM et al., [9]. Kridis WB et al., [14] have reported a case of cerebral metastasis from Mandibular gingiva. Our case is the second of its kind, to metastasise to brain from mandibular gingiva (Table-1).

Currently, Union for International Cancer Control (UICC) and the American Joint Committee on Cancer (AJCC) developed staging system for mucosal melanoma of head and neck. This reflects the aggressivebehavior and the classification for mucosal melanoma of head and neck, begins with stage III [6, 8]. According to which our patient was classified under Stage IVC.

There are no guidelines available for the management of OMM. Wide surgical resection of the primary lesion with Elective neck dissection and adjuvant postoperative radiotherapy to maximize locoregional controlremains the mainstay of therapeutic approach [8].Adjuvant Chemotherapy [Cisplatin, vinblastine sulfate, dacarbazine]does not improve survival and mainly used for palliative purpose [4, 8]. Immunotherapy [interleukin-2, interferon (IFN)-a-2b, imatinib, and paclitaxel] and bio-chemotherapy [combination of cytotoxic and immunomodulatory regimens] have been significantly correlated with improved survival in patients with advanced and metastatic disease [8]. The US Food and Drug Administration (FDA) approved checkpoint inhibitors vemurafenib (BRAFinhibitor) ipilimumab (anti-CTLA4) and nivolumab and pembrolizumab (anti-PD-1) for use as first-line therapy for metastatic melanoma and are in clinical phase trials [8]. In our case, patient reported to us, in Stage 1VC with cerebral metastasis and the treatment option planned was palliative therapy.

Regardless of multimodality therapeutic approaches, prognosis of OMMremains poor with 5 year survival rate of 10-25\% forearly-stage disease and $0 \%$ for patients with advanced-stage tumors, regional and/or distant metastasis [8]. Death usually results from distant metastasis rather than lack of local control. Brain metastasis contributes to death in nearly $95 \%$ of patients with median survival rate of less than a year despite treatment [13]. The worst prognosis of OMM may be due to late diagnosis, different histopathological behavior, tendency to deep invasion, early hematogenous metastases, anatomical peculiarities of the region with difficulty in the surgical resection with disease-free margins and the absence of standardized treatment protocols [5]. In our case, there was invasion into adjacent alveolar bone, regional sub-mandibular node and distant cerebral metastasiswith poor prognosis and $0 \%$ survival rate.

\section{CONCLUSION}

Melanoma is aexceedingly rare malignant tumor of the oral cavity and is often reported and diagnosed at their advanced stages with regional and distant metastasis primarily due to their asymptomatic nature in the early stages of the lesion.Owing to the aggressive nature, early diagnosis and treatment is crucial for better survival and prognosis.To achieve this, it is necessary to perform a more detailed oral mucosa examination and biopsies of all macular or nodular suspectedpigmented lesions. Thus dental surgeons play a key role in the early diagnosis of oral melanoma.

\section{REFERENCE}

1. Meleti, M., Leemans, C. R., Mooi, W. J., Vescovi, P., \& van der Waal, I. (2007). Oral malignant melanoma: a review of the literature. Oral oncology, 43(2), 116-121.

2. Femiano, F., Lanza, A., Buonaiuto, C., Gombos, F., Di Spirito, F., \& Cirillo, N. (2008). Oral malignant melanoma: a review of the literature. Journal of oral pathology \& medicine, 37(7), 383-388.

3. Prasad, L. K., Chakravarthi, P. S., Devi, K. N. N., Sridhar, M., Kumar, Y. R., \& Yesuratnam, D. (2009). Malignant melanoma of lingual gingiva with ipsilateral submandibular lymph node metastasis: a case report. Journal of maxillofacial and oral surgery, 8(3), 287-289.

4. Tanaka, N., Mimura, M., Ogi, K., \& Amagasa, T. (2004). Primary malignant melanoma of the oral cavity: assessment of outcome from the clinical records of 35 patients. International journal of oral and maxillofacial surgery, 33(8), 761-765.

5. Guevara-Canales, J. O., Gutiérrez-Morales, M. M., Sacsaquispe-Contreras, S. J., Sánchez-Lihón, J., \& Morales-Vadillo, R. (2012). Malignant melanoma of the oral cavity. Review of the literature and experience in a Peruvian Population. Medicina oral, patologia oral y cirugia bucal, 17(2), e206211.

6. Warszawik-Hendzel, O., Słowińska, M., Olszewska, M., \& Rudnicka, L. (2014). Melanoma of the oral cavity: pathogenesis, dermoscopy, clinical features, staging and management. Journal of dermatological case reports, 8(3), 60-66.

7. Gauwerky, K. J., Ehrenfeld, M., Bakos, R. M., Volkenandt, M., Ruzicka, T., \& Berking, C. (2010). A rare case of local relapsing oral 
melanoma. JDDG: Journal der Deutschen Dermatologischen Gesellschaft, 8(8), 614-616.

8. Chatzistefanou, I., Kolokythas, A., Vahtsevanos, K., \& Antoniades, K. (2016). Primary mucosal melanoma of the oral cavity: current therapy and future directions. Oral surgery, oral medicine, oral pathology and oral radiology, 122(1), 17-27.

9. Tlholoe, M. M., Khammissa, R. A. G., Bouckaert, M., Altini, M., Lemmer, J., \& Feller, L. (2015). Oral mucosal melanoma: some pathobiological considerations and an illustrative report of a case. Head and neck pathology, 9(1), 127-134.

10. Greene, G. W., Haynes, J. W., Dozier, M., Blumberg, J. M., \& Bernier, J. L. (1953). Primary malignant melanoma of the oral mucosa. Oral Surgery, Oral Medicine, Oral Pathology, 6(12), 1435-1443.

11. Barker, B. F., Carpenter, W. M., Daniels, T. E., Kahn, M. A., Leider, A. S., Lozada-Nur, F., ... \& Peters, E. (1997). Oral muscosal melanomas: the
WESTOP Banff workshop proceedings. Oral Surgery, Oral Medicine, Oral Pathology, Oral Radiology, and Endodontology, 83(6), 672-679.

12. Martínez, E. Á., Alonso, F. C., Siles, M. S., \& Jornet, P. L. (2005). Melanoma of the oral mucosa with cerebral metastasis: a clinical case. Oral Oncology Extra, 41(2), 30-33.

13. Sloan, A. E., Nock, C. J., \& Einstein, D. B. (2009). Diagnosis and Treatment of mela-noma Brain Metastasis: A Literature Review. Cancer Control, 16(3), 248-255.

14. Kridis, W. B., Feki, J., Ayedi, L., Khanfir, A., Toumi, N., Abdelmoula, M., ... \& Frikha, M. (2016). Primary Gingival Melanoma: An Important Entity. Journal of maxillofacial and oral surgery, 15(2), 287-290.

15. Sun, C. Z., Chen, Y. F., Jiang, Y. E., Hu, Z. D., Yang, A. K., \& Song, M. (2012). Treatment and prognosis of oral mucosal melanoma. Oral oncology, 48(7), 647-652. 